\title{
Mechanischer Ileus auf der Basis eines Phytobezoars
}

\section{Einleitung}

Bezoare sind mit einer Inzidenz von <0,001 (Iwamuro et al. World ] Gastrointest Endosc 2015; 7: 336 - 345) klinisch selten auftretende Konglomerate unverdaulicher Materialien, die mit der Nahrung aufgenommen werden. Die häufigste Form ist der Phytobezoar, bestehend aus unverdaulichem Pflanzenmaterial (Iwamuro et al. World J Gastrointest Endosc 2015; 7: 336 -345). Sie können jahrelang symptomfrei im Magen-Darm-Trakt verweilen und bilden sich zumeist auf dem Boden einer verzögerten gastrointestinalen Passage. Schätzungsweise $90 \%$ gehen symptomlos über den Verdauungstrakt ab. Stenosieren oder verlegen sie jedoch das Lumen, besonders häufig am Magenausgang, führen sie zu Symptomen bis hin zum akuten Abdomen bei Ausbildung eines mechanischen lleus. Damit sind sie für etwa 0,4-4\% aller mechanischen Ilei verantwortlich (Dikicier et al. World J Clin Cases 2015; 3: 721 - 726).

\section{Fallbeschreibung}

Eine 36-jährige Patientin aus einem Flüchtlingsheim wird mit seit 3 Tagen anhaltender Übelkeit, Erbrechen und krampfartigen Oberbauchschmerzen über die Notaufnahme auf eine gastroenterologische Normalstation aufgenommen. Bei unauffälligen Infektparametern sowie Stuhlverhalt und fehlendem abdominellem Druckschmerz oder tastbaren Resistenzen ist initial nicht von einem Magen-DarmVirus oder einer Appendizitis auszugehen. Auch eine initial in der Notaufnahme durchgeführte Sonografie zeigt keinen pathologischen Befund. Aus der Vorgeschichte der Patientin sind aufgrund bestehender Sprachbarrieren wenige anamnestische Details bekannt. Sie leidet unter Zöliakie Typ III B nach Marsh, die Milz wurde aufgrund einer Milzvenenthrombose mittels medianer Laparotomie entnommen; es zeigt sich eine großflächige, reizlose Narbenhernie ohne Hinweis auf Inkarzeration.

Initial wird zum Ausschluss eines Ileus oder einer Darmperforation ein Röntgen des Abdomens im Stehen angefertigt. Bereits auf ihm ist sehr flau eine inhomogene

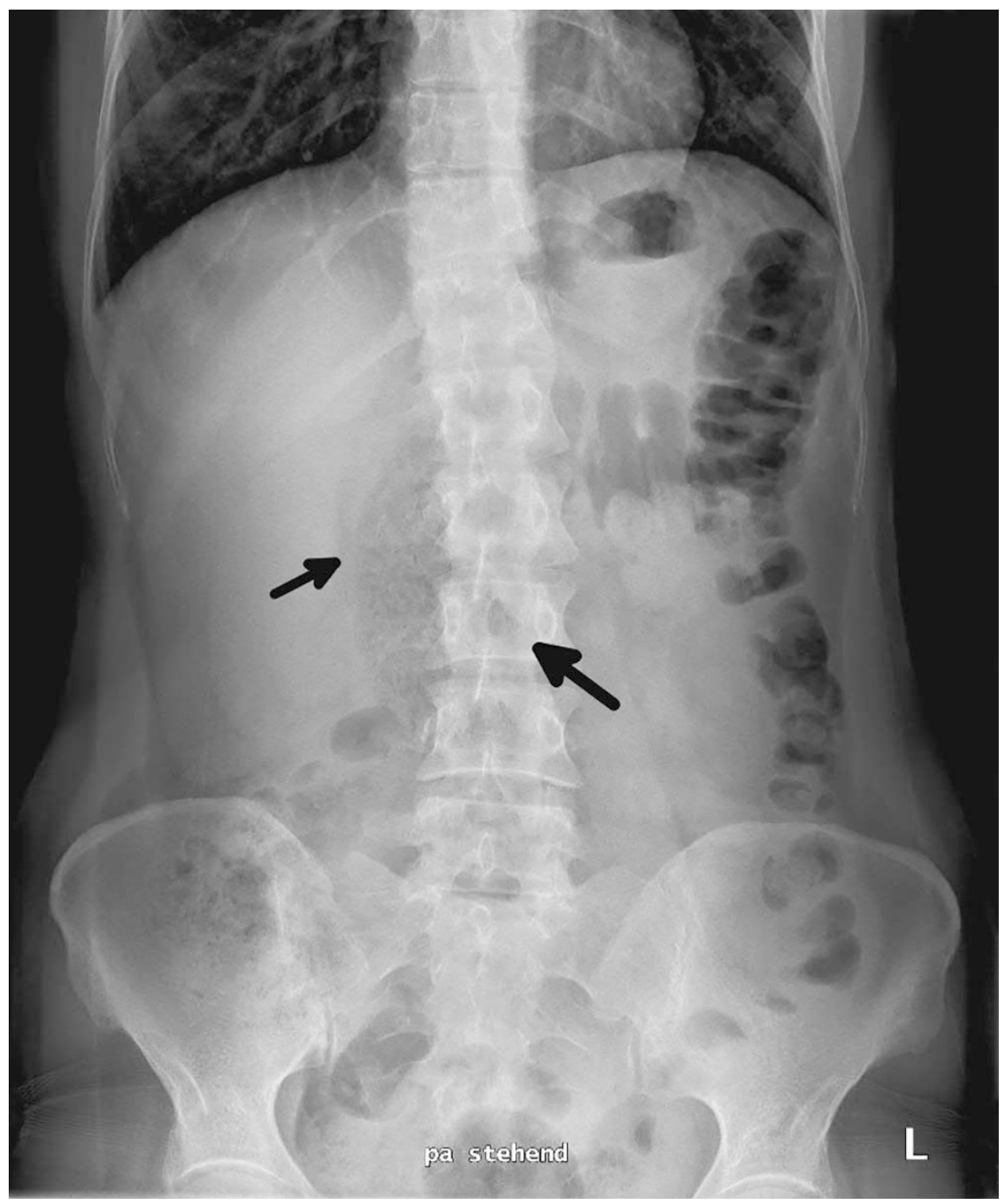

- Abb. 1 Abdomen p. a. stehend. Die initiale Röntgenaufnahme ermöglicht den Ausschluss freier intraabdomineller Luft und zeigt den inhomogenen Fremdkörper in Projektion auf die Lendenwirbelsäule.

Masse abgrenzbar ( $\triangleright$ Abb. 1), die als Koprostase gewertet wird. Bei regredienter Symptomatik und der Verdachtsdiagnose Gastritis wird eine Oesophago-Gastro-Duodenoskopie (ÖGD) durchgeführt, die initial aufgrund des mit Nahrung gefüllten Magens abgebrochen werden muss. Es zeigen sich eine Antrumgastritis und eine Duodenitis. Im durchgeführten CT-Abdomen ist ein Fremdkörper von inhomogener Dichte abgrenzbar, der aber im radiologischen Befund keine Erwähnung findet und als Koprostase verkannt wird ( $\triangleright$ Abb. $2 \mathbf{a}-\mathbf{c}$ ). Unter Anlage einer Magensonde sind die Beschwerden der Patientin deutlich rückläufig. Die drei Tage später erfolgende Kon- trollgastroskopie erbringt den Nachweis eines gelblichen, inhomogenen Materials im Bereich des Duodenums, das seitlich nicht passierbar ist. Der Versuch, den Fremdkörper mittels Extraktionsschlinge und Dormiakörbchen zu bergen, schlägt fehlt, sodass nach Probengewinnung die ÖGD abgebrochen wird ( $\triangleright$ Abb. 3). Zusätzlich ergibt sich bei narbigen Formationen im Bereich des Magenausgangs der Verdacht, dass die Patientin in ihrem Heimatland einer Magenoperation unterzogen wurde, am ehesten einer Magenteilresektion. Der Pylorus zeigt sich weit eröffnet und der klinisch bestehende Verdacht einer distalen Gastroparese bestätigt sich endoskopisch. 

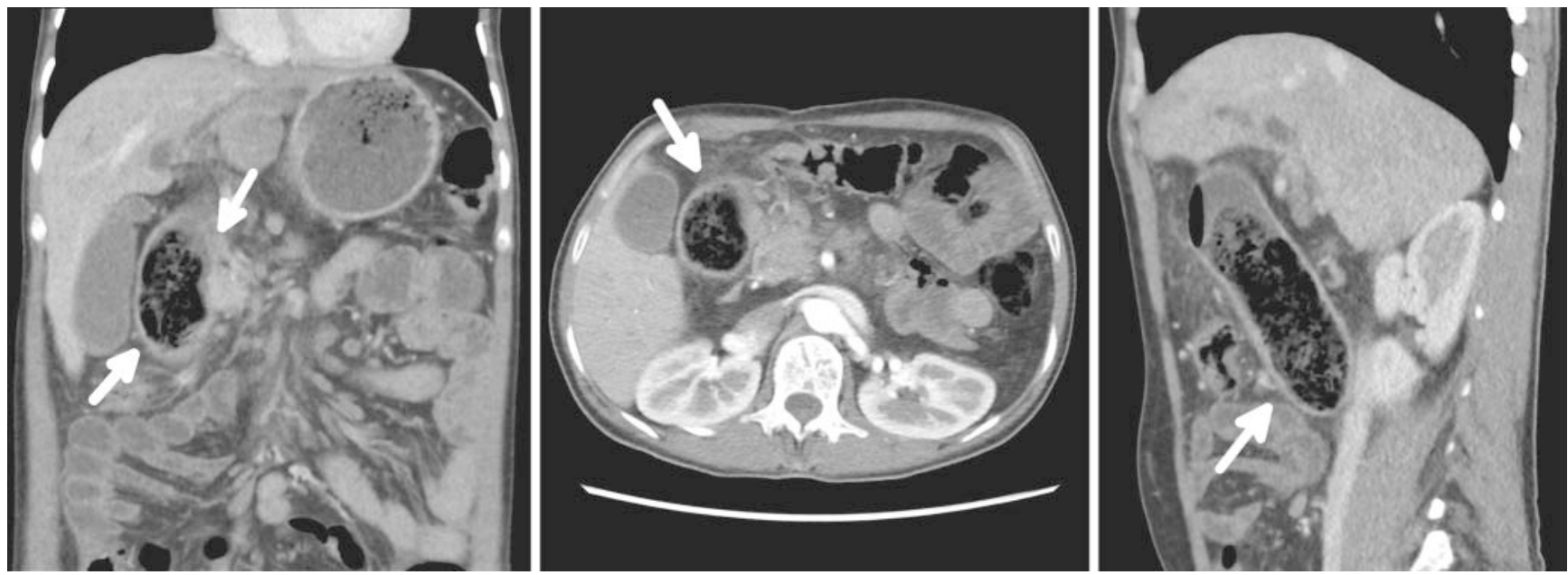

- Abb. 2 a-c CT Abdomen mit KM, arterielle Phase, Weichteilfenster. In der CT ist eine dichteinhomogene, ca. $12 \mathrm{~cm} \times 4 \mathrm{~cm} \times 4 \mathrm{~cm}$ messende, glatt begrenzte Masse in der Pars descendens und inferior duodeni abgrenzbar, die zur Aufdehnung des Lumens führt und ein Passagehindernis darstellt. Die Binnenstruktur ähnelt typischerweise der von Koprostase, was Verwechselungsgefahr hervorruft. Das Duodenum umgebendes Fettgewebe weist Entzündungszeichen auf.

Im Lauf des Tages wird eine MagenDarm-Passage mittels jodhaltigen Kontrastmittels durchgeführt. Dabei zeigt sich ein geringer Fortschritt des Fremdkörpers, der von proximal von Kontrastmittel umschlossen wird ( $\triangleright$ Abb.4a-d).

Im Verlauf entwickelt die Patientin progrediente Ileussymptomatik und wird nach prolongiertem Verlauf auf die Viszeralchirurgie verlegt. Der Bezoar wird noch am selben Tag per medianer Laparotomie und lokaler Ileotomie geborgen. Zusätzlich fanden mehrfache Adhäsiolysen statt. Die histologische Analyse des gesamten gewonnenen Materials zeigt eine primäre Zusammensetzung aus unverdaulichem pflanzlichem Material, es ergibt sich die Diagnose eines Phytobezoars.

Der weitere Verlauf gestaltet sich unkompliziert, die Patientin kann binnen weniger Tage entlassen werden.

\section{Diskussion}

Bereits zum Aufnahmezeitpunkt zeigte die Patientin die typische Klinik eines Ileus. Die Abdomenübersichtsaufnahme zeigte bereits den Fremdkörper, dieser wurde jedoch als Koprostase in der rechten Kolonflexur gedeutet - ein klassischer Fehler.

Die Abdomen-CT hat in der Diagnostik von Bezoaren den höchsten Stellenwert, da Lokalisation und Größe des Fremdkörpers beschrieben und synchron auftretende Bezoare erkannt werden können. Im beschrie-

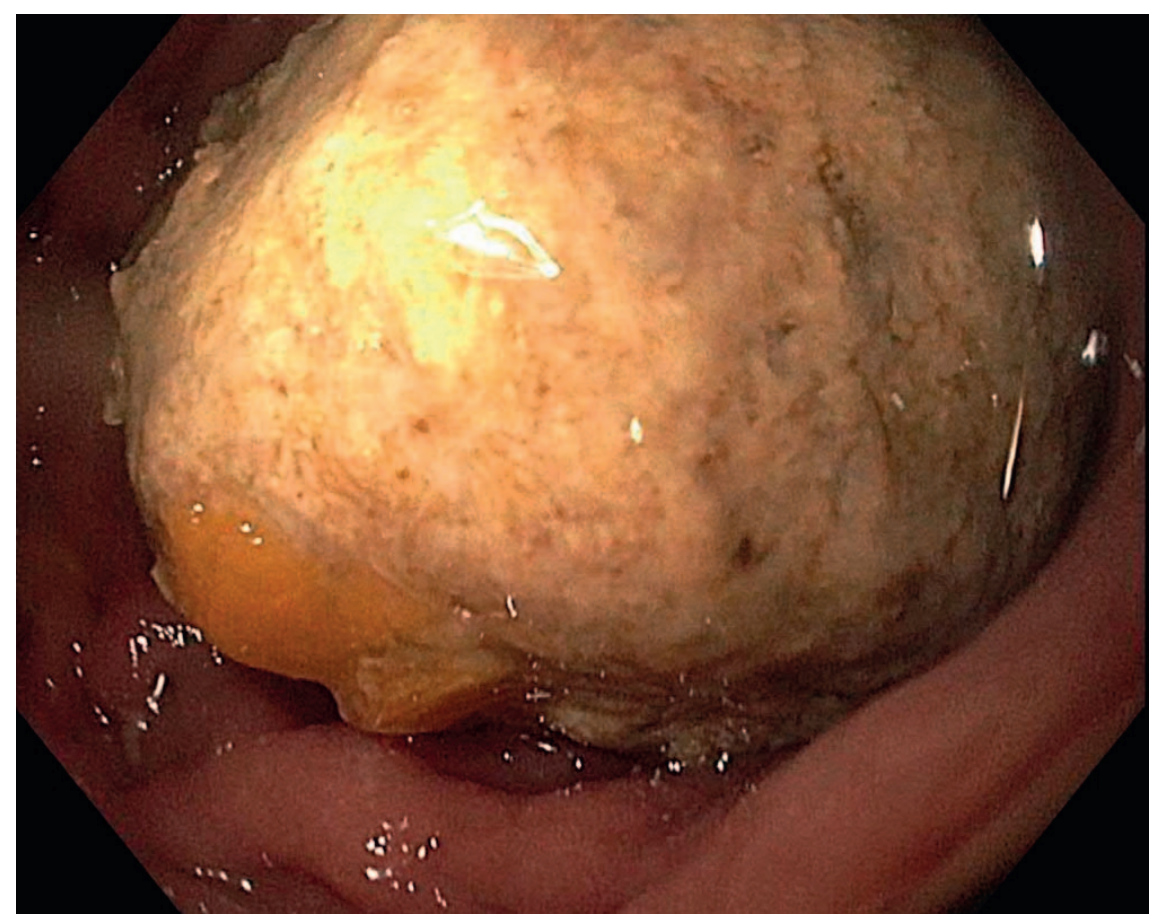

- Abb. 3 Endoskopische Aufnahme bei Ösophago-Gastro-Duodenoskopie. In der zweiten durchgeführten ÖGD lässt sich ein gelblicher, inhomogener Fremdkörper darstellen, der im Bereich des distalen Duodenums zu liegen kommt und nicht passierbar ist.

benen Fall hätte bereits die initiale Abdomen-CT ausgereicht, über die weitere Therapie zu entscheiden; der Bezoar wurde aber nicht erkannt, sodass ein konservatives Vorgehen favorisiert und weitere Ursachensuche durch eine ÖGD betrieben wurde.

Neben der Diagnostik bietet die ÖGD den Vorteil, verschiedene Techniken zur minimalinvasiven Therapie zu ermöglichen. Im beschriebenen Fall entschied man sich trotz fehgeschlagenen Bergungsversuchs weiterhin zur konservativen Therapie bei regredienter Klinik. Die additive Durchführung einer MagenDarm-Passage mit jodhaltigem Kontrastmittel ist aus klinischer Sicht nicht nach- 

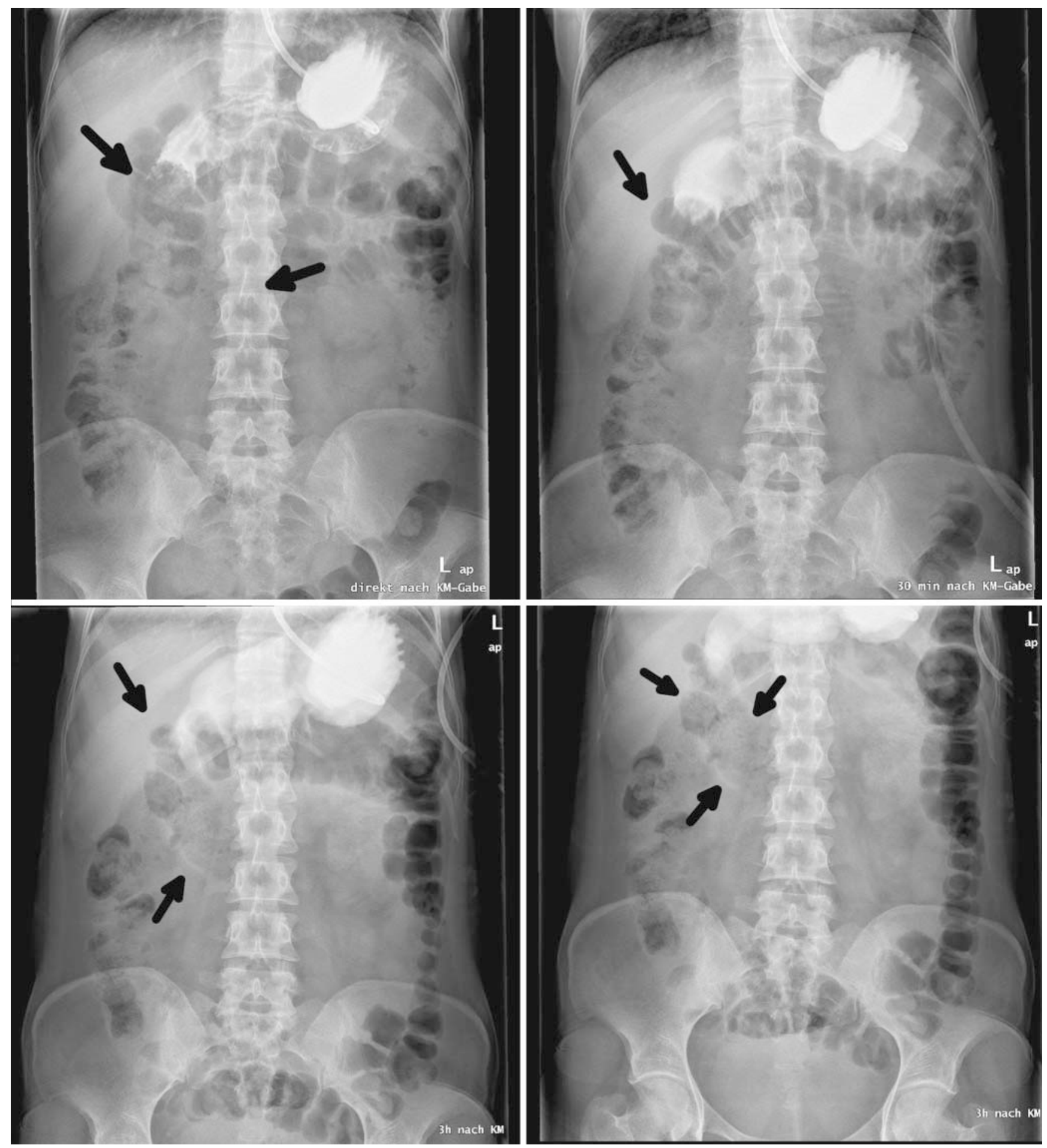

- Abb. 4 a-d a Abdomen a. p. direkt nach Kontrastmittelgabe über die Magensonde. In der Magen-Darm-Passage zeigt sich bereits initial eine gute Kontrastierung des Magens bis in das Duodenum hinein. b Abdomen a. p. 30 Minuten nach Kontrastmittelgabe. Es zeigt sich immer stärker ein unscharf abgrenzbares Hindernis im Duodenum, das kappenartig umspült wird. Die Magensonde der Patientin wird zum Schutz vor Aspiration geöffnet. c Abdomen a. p. 3 Stunden nach Kontrastmittelgabe. Durch die Öffnung der Magensonde fließt das Kontrastmittel ab, die Kontrastierung des Magens nimmt ab. Zusätzlich lässt sich nun erkennen, wie kleine Mengen Kontrastmittel den duodenalen Fremdkörper in proximalen Anteilen umspülen (Pfeil). d Abdomen a. p. 6 Stunden nach Kontrastmittelgabe. Sehr flau zeigt sich eine Kontrastierung distalerer Dünndarmanteile. Geringe Mengen des Kontrastmittels können am Bezoar vorbei transportiert werden. 
vollziehbar, da der Bezoar als Verursacher des mechanischen Ileus bereits bekannt war und daher kein zusätzlicher Informationsgewinn zu erwarten war. Stattdessen ist hier das Risiko einer Aspiration von Kontrastmittel bei rezidivierendem Erbrechen imminent, die Untersuchung war kontraindiziert.

Eine chirurgische Therapie, etwa durch die in diesem Fall angewendete Ileotomie, hätte deutlich eher in Betracht gezogen werden müssen, da die Bergungs- und Zerkleinerungsversuche mittels Gastroskopie erfolglos blieben, obwohl das Potenzial nicht vollständig ausgeschöpft war. So hätte ein Lyseversuch mittels Coca-Cola ${ }^{\circledR}$ (Ladas et al. Aliment Pharmacol Ther 2013; 37: 169 -173), Papain oder Cellulase in Verbindung mit einer Endoskopie (Iwamuro et al. World J Gastrointest Endosc 2015; 7: 336 - 345) stattfinden können.

\section{KERNAUSSAGEN:}

- Bezoare als wichtige, aber seltene Differenzialdiagnose bei Verdacht auf mechanischen lleus

- Prädisponierend sind Paresen des Gastrointestinaltrakts insbesondere nach abdominalchirugischen Eingriffen

- CT-Scan als Mittel der Wahl zur Darstellung von Lage und Größe des Bezoars sowie zum Auffinden von Zweitbezoaren

- Magen-Darm-Passage aufgrund der Komplikationsrate eher zurückhaltend zu verwenden bis kontraindiziert

\section{Interessenkonflikt}

Die Autoren geben an, dass kein Interessenkonflikt besteht.
Autorinnen/Autoren

Stephanie Gräger, Ulf Teichgräber

Institute of Diagnostic and Interventional Radiology, University Hospital Jena, Germany

Korrespondenzadresse

Miss Stephanie Gräger

Institute of interventional and diagnostic

Radiology, University Hospital Jena

Am Klinikum 1

07747 Jena

07743 Jena

Germany

Tel.: ++49/3641/9324856

stephanie.graeger@med.uni-jena.de

Bibliografie

DOI https://doi.org/10.1055/a-0690-9292 Online-Publikation: 4.9.2018

Fortschr Röntgenstr 2019; 191: 228-231

(c) Georg Thieme Verlag KG, Stuttgart · New York ISSN 1438-9029 